\title{
Erratum
}

\section{Genome Size Variation in Malus Species}

\author{
Monika Höfer ${ }^{1}$ and Armin Meister ${ }^{2}$ \\ ${ }^{1}$ Julius Kuehn-Institute, Federal Research Centre for Cultivated Plants, Institute for Breeding Research on Horticultural and Fruit Crops, \\ Pillnitzer Platz 3a, 01326 Dresden, Germany \\ ${ }^{2}$ Leibniz-Institut für Pflanzengenetik und Kulturpflanzenforschung Gatersleben (IPK), Corrensstraße 3, D. 06466 Gatersleben, \\ Germany
}

Correspondence should be addressed to Monika Höfer, monika.hoefer@jki.bund.de

Received 18 August 2010; Accepted 24 August 2010

Copyright (C) 2010 M. Höfer and A. Meister. This is an open access article distributed under the Creative Commons Attribution License, which permits unrestricted use, distribution, and reproduction in any medium, provided the original work is properly cited.

The sectional classification of the genus Malus should correctly read Eumalus (Malus).

In the Introduction section, the third paragraph should read "Most species are diploid $(x=17)$ and cross-pollinated, but Way et al. [10] list Malus coronaria from the American section Chloromeles as apomictic and either triploid or tetraploid. They also note deviations from the normal type in section Eumalus and Sorbomalus: M. sikkimensis, $M$. hupehensis and M. toringoides (apomictic and triploid), M. sargenti (apomictic and tetraploid), and M. spectabilis and M. baccata (nonapomictic and either diploid or tetraploid)," while the sixth paragraph in the Discussion section should be written as "Harris et al. [30] analysed nuclear rDNA and chloroplast DNA sequences and placed Malus trilobata and Malus florentina in the same cluster. The present measurement of the DNA content would support the results of Harris et al. [30]. Qian et al. [7] characterized Malus florentina as a new section Florentinae. 25 species including 17 hybrid species demonstrated 1Cx DNA contents in the interval between $-5 \%$ and $+5 \%$ of the median of the diploid species. All species belong to the section Eumalus and Sorbomalus, series Sieboldianae and Kansuenses, and have native distribution in Asia and Europe with the exception of the species M. fusca spread on the West coast of North America." Finally, the word Euromalus should be changed into Eumalus in all the occasions mentioned in Table 1 as well. 
TABle 1: Nuclear DNA content (pg/2C median; 25\% and 75\% value) of Malus species (Number of accessions used for measurements) with the results of the nonparametric Kruskal-Wallis Test together with Dunn's Multiple Range Test; medians with the same letter was not significantly different at 5\% level. Each ploidy level was analysed separately. Classification was based on Rehder [5] and Langenfelds [14].

\begin{tabular}{|c|c|c|c|c|c|c|c|c|c|c|c|c|}
\hline \multirow{2}{*}{ Species } & \multirow{2}{*}{ Section } & \multirow{2}{*}{ Series } & \multicolumn{4}{|c|}{ Nuclear DNA content (pg/nucleus) } & \multicolumn{6}{|c|}{ Results of the Kruskal-Wallis test } \\
\hline & & & No. & Median* & $25 \%$ & $75 \%$ & & & & & & \\
\hline Ploidy level $2 x=34$ & & & & 1.484 & & & & & & & & \\
\hline M. tschonoskii & Docyniopsis & & 1 & 1.245 & 1.150 & 1.283 & a & & & & & \\
\hline M. yunnanensis & Sorbomalus & Yunnanenses & 2 & 1.337 & 1.320 & 1.350 & $\mathrm{a}$ & & & & & \\
\hline M. ombrophila & Sorbomalus & Yunnanenses & 1 & 1.341 & 1.329 & 1.351 & a & $\mathrm{b}$ & & & & \\
\hline M. kansuensis & Sorbomalus & Kansuenses & 6 & 1.365 & 1.350 & 1.384 & $\mathrm{a}$ & $\mathrm{b}$ & & & & \\
\hline M. honanensis & Sorbomalus & Yunnanenses & 1 & 1.378 & 1.276 & 1.413 & $\mathrm{a}$ & $\mathrm{b}$ & & & & \\
\hline M. prattii & Sorbomalus & Yunnanenses & 1 & 1.397 & 1.386 & 1.406 & $\mathrm{a}$ & $\mathrm{b}$ & & & & \\
\hline M. baccata & Eumalus & Baccatae & 16 & 1.440 & 1.430 & 1.467 & & $\mathrm{~b}$ & & & & \\
\hline M. $\times z u m i ~ 2 x$ & Eumalus & Baccatae & 10 & 1.461 & 1.439 & 1.512 & & $\mathrm{~b}$ & c & & & \\
\hline M. $\times$ dawsoniana & Sorbomalus & Kansuenses & 1 & 1.464 & 1.455 & 1.478 & & $\mathrm{~b}$ & c & & & \\
\hline M. fusca & Sorbomalus & Kansuenses & 5 & 1.464 & 1.460 & 1.470 & & $\mathrm{~b}$ & c & & & \\
\hline M. hupehensis $2 \mathrm{x}$ & Eumalus & Baccatae & 2 & 1.466 & 1.451 & 1.475 & & $\mathrm{~b}$ & c & & & \\
\hline M. $\times$ hartwigii & Eumalus & Baccatae & 1 & 1.471 & 1.450 & 1.475 & & $\mathrm{~b}$ & c & & & \\
\hline M. $\times$ adstringensis $2 \mathrm{x}$ & Eumalus & Baccatae & 5 & 1.475 & 1.454 & 1.493 & & $\mathrm{~b}$ & c & & & \\
\hline M. $\times$ moerlandsii & Eumalus & Pumilae & 4 & 1.476 & 1.457 & 1.493 & & $\mathrm{~b}$ & c & & & \\
\hline M. ×purpurea & Eumalus & Pumilae & 7 & 1.484 & 1.467 & 1.493 & & & c & $\mathrm{d}$ & & \\
\hline M. sylvestris & Eumalus & Pumilae & 11 & 1.484 & 1.460 & 1.509 & & & c & $\mathrm{d}$ & & \\
\hline M. $\times$ robusta & Eumalus & Baccatae & 5 & 1.490 & 1.458 & 1.519 & & & c & $\mathrm{d}$ & & \\
\hline M. $\times$ atrosanguinea & Eumalus & Baccatae & 1 & 1.493 & 1.449 & 1.493 & & $\mathrm{~b}$ & c & $\mathrm{d}$ & & \\
\hline M. $\times$ sublobata & Eumalus & Pumilae & 4 & 1.497 & 1.459 & 1.550 & & & c & $\mathrm{d}$ & & \\
\hline M. $\times$ arnoldiana & Eumalus & Baccatae & 3 & 1.499 & 1.470 & 1.529 & & & c & $\mathrm{d}$ & & \\
\hline M. $\times$ asiatica & Eumalus & Pumilae & 4 & 1.500 & 1.470 & 1.514 & & & c & $\mathrm{d}$ & & \\
\hline M. prunifolia & Eumalus & Pumilae & 10 & 1.500 & 1.475 & 1.519 & & & c & $\mathrm{d}$ & & \\
\hline M. sieversii & Eumalus & Pumilae & 6 & 1.501 & 1.482 & 1.513 & & & c & $\mathrm{d}$ & & \\
\hline M. orientalis & Eumalus & Pumilae & 7 & 1.502 & 1.458 & 1.519 & & & c & $\mathrm{d}$ & & \\
\hline M. spectabilis $2 \mathrm{x}$ & Eumalus & Pumilae & 7 & 1.502 & 1.484 & 1.523 & & & c & $\mathrm{d}$ & e & \\
\hline M. $\times$ scheideckeri & Sorbomalus & Sieboldianae & 3 & 1.507 & 1.479 & 1.530 & & & c & $\mathrm{d}$ & e & \\
\hline M. floribunda & Sorbomalus & Sieboldianae & 6 & 1.509 & 1.490 & 1.529 & & & c & $\mathrm{d}$ & $\mathrm{e}$ & \\
\hline M. $\times$ domestica $2 \mathrm{x}$ & Eumalus & Pumilae & 8 & 1.514 & 1.487 & 1.530 & & & c & $\mathrm{d}$ & e & \\
\hline M. $\times$ micromalus & Eumalus & Baccatae & 2 & 1.528 & 1.497 & 1.534 & & & c & $\mathrm{d}$ & e & \\
\hline M. sieboldii $2 \mathrm{x}$ & Sorbomalus & Sieboldianae & 5 & 1.530 & 1.505 & 1.533 & & & & $\mathrm{~d}$ & e & \\
\hline M. transitoria & Sorbomalus & Kansuenses & 5 & 1.535 & 1.514 & 1.551 & & & & $\mathrm{~d}$ & e & \\
\hline M. $\times$ soulardii & Chloromeles & & 4 & 1.564 & 1.512 & 1.588 & & & & $\mathrm{~d}$ & e & $\mathrm{f}$ \\
\hline M. ioensis $2 \mathrm{x}$ & Chloromeles & & 4 & 1.565 & 1.523 & 1.667 & & & & & $\mathrm{e}$ & $\mathrm{f}$ \\
\hline M. trilobata & Eriolobus & & 3 & 1.638 & 1.621 & 1.667 & & & & & & $\mathrm{f}$ \\
\hline M. florentina & Sorbomalus & Florentinae & 3 & 1.653 & 1.635 & 1.660 & & & & & & $\mathrm{f}$ \\
\hline Ploidy level 3x $=51$ & & & & 2.198 & & & & & & & & \\
\hline M. hupehensis 3x & Eumalus & Baccatae & 18 & 2.121 & 2.014 & 2.174 & $\mathrm{a}$ & & & & & \\
\hline M. sikkimensis 3x & Eumalus & Baccatae & 7 & 2.165 & 2.148 & 2.180 & $\mathrm{a}$ & $\mathrm{b}$ & & & & \\
\hline M. $\times$ zumi $3 \mathrm{x}$ & Eumalus & Baccatae & 1 & 2.194 & 2.143 & 2.229 & $\mathrm{a}$ & $\mathrm{b}$ & c & & & \\
\hline M. sieboldii 3x & Sorbomalus & Sieboldianae & 3 & 2.193 & 2.183 & 2.201 & & $\mathrm{~b}$ & c & & & \\
\hline M. $\times$ adstring $3 \mathrm{x}$ & Eumalus & Baccatae & 3 & 2.201 & 2.191 & 2.211 & & $\mathrm{~b}$ & c & & & \\
\hline M. toringoides $3 \mathrm{x}$ & Sorbomalus & Kansuenses & 7 & 2.202 & 2.172 & 2.254 & & $\mathrm{~b}$ & c & & & \\
\hline M. spectabilis $3 \mathrm{x}$ & Eumalus & Pumilae & 1 & 2.206 & 2.195 & 2.216 & & $\mathrm{~b}$ & c & & & \\
\hline M. halliana & Eumalus & Baccatae & 3 & 2.209 & 2.174 & 2.217 & & $\mathrm{~b}$ & c & & & \\
\hline M. komarovii 3x & Sorbomalus & Kansuenses & 5 & 2.232 & 2.210 & 2.261 & & & c & $\mathrm{d}$ & & \\
\hline
\end{tabular}


TABle 1: Continued.

\begin{tabular}{|c|c|c|c|c|c|c|c|c|c|c|c|}
\hline \multirow{2}{*}{ Species } & \multirow{2}{*}{ Section } & \multirow{2}{*}{ Series } & \multicolumn{4}{|c|}{ Nuclear DNA content (pg/nucleus) } & \multicolumn{5}{|c|}{ Results of the Kruskal-Wallis test } \\
\hline & & & No. & Median* & $25 \%$ & $75 \%$ & & & & & \\
\hline M. $\times$ domestica $3 \mathrm{x}$ & Eumalus & Pumilae & 6 & 2.270 & 2.252 & 2.296 & & & c & $\mathrm{d}$ & \\
\hline M. sargenti $3 \mathrm{x}$ & Sobomalus & Sieboldianae & 2 & 2.285 & 2.197 & 2.307 & & & & d & \\
\hline M. ioensis $3 \mathrm{x}$ & Chloromeles & & 2 & 2.414 & 2.396 & 2.426 & & & & d & \\
\hline M. coronaria $3 \mathrm{x}$ & Chloromeles & & 3 & 2.428 & 2.183 & 2.462 & & & & $\mathrm{~d}$ & \\
\hline Ploidy level $4 \mathrm{x}=68$ & & & & 3.007 & & & & & & & \\
\hline M. sikkimensis $4 \mathrm{x}$ & Eumalus & Baccatae & 2 & 2.851 & 2.815 & 2.894 & $\mathrm{a}$ & & & & \\
\hline M. sieboldii 4x & Sorbomalus & Sieboldianae & 5 & 2.879 & 2.832 & 2.938 & $\mathrm{a}$ & & & & \\
\hline M. komarovii 4x & Sorbomalus & Kansuenses & 2 & 2.917 & 2.847 & 3.013 & $\mathrm{a}$ & $\mathrm{b}$ & & & \\
\hline M. sargenti $4 \mathrm{x}$ & Sorbomalus & Sieboldianae & 4 & 2.918 & 2.891 & 2.958 & $\mathrm{a}$ & $\mathrm{b}$ & & & \\
\hline M. toringoides $4 \mathrm{x}$ & Sorbomalus & Kansuenses & 1 & 2.958 & 2.947 & 2.979 & $\mathrm{a}$ & $\mathrm{b}$ & c & & \\
\hline M. lancifolia & Chloromeles & & 1 & 3.007 & 2.969 & 3.035 & & & c & $\mathrm{d}$ & \\
\hline M. glaucescens & Chloromeles & & 1 & 3.102 & 3.082 & 3.197 & & & & $\mathrm{~d}$ & $\mathrm{e}$ \\
\hline M. angustifolia & Chloromeles & & 1 & 3.116 & 3.101 & 3.122 & & & c & $\mathrm{d}$ & $\mathrm{e}$ \\
\hline M. $\times$ platycarpa & Chloromeles & & 4 & 3.151 & 3.118 & 3.177 & & & & $\mathrm{~d}$ & $\mathrm{e}$ \\
\hline M. coronaria $4 \mathrm{x}$ & Chloromeles & & 6 & 3.186 & 3.115 & 3.221 & & & & & $\mathrm{e}$ \\
\hline M. $\times$ heterophylla & Chloromeles & & 1 & 3.193 & 3.167 & 3.216 & & & & & $\mathrm{e}$ \\
\hline \multicolumn{12}{|l|}{ Ploidy level 5x-6x } \\
\hline M. sikkimensis $5 \mathrm{x}$ & Eumalus & Baccatae & 1 & 3.540 & & & & & & & \\
\hline M. sargenti $5 \mathrm{x}$ & Sorbomalus & Sieboldianae & 1 & 3.874 & & & & & & & \\
\hline M. sargenti $5 \mathrm{x}-6 \mathrm{x}$ & Sorbomalus & Sieboldianae & 1 & 4.286 & & & & & & & \\
\hline
\end{tabular}

${ }^{*}$ Estimation based on propidium iodide staining by using the internal standard radish (1.1 pg/2C). 

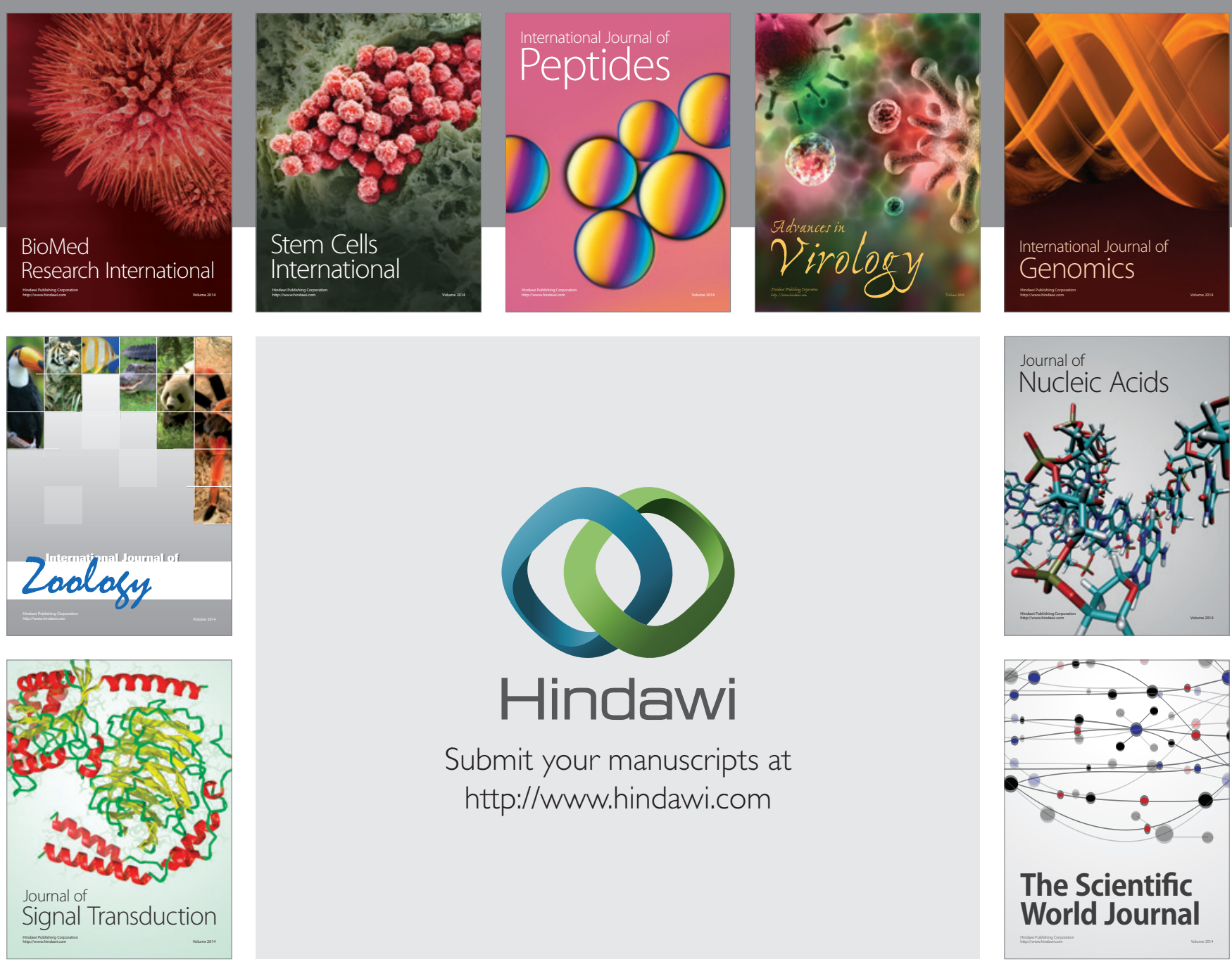

Submit your manuscripts at

http://www.hindawi.com
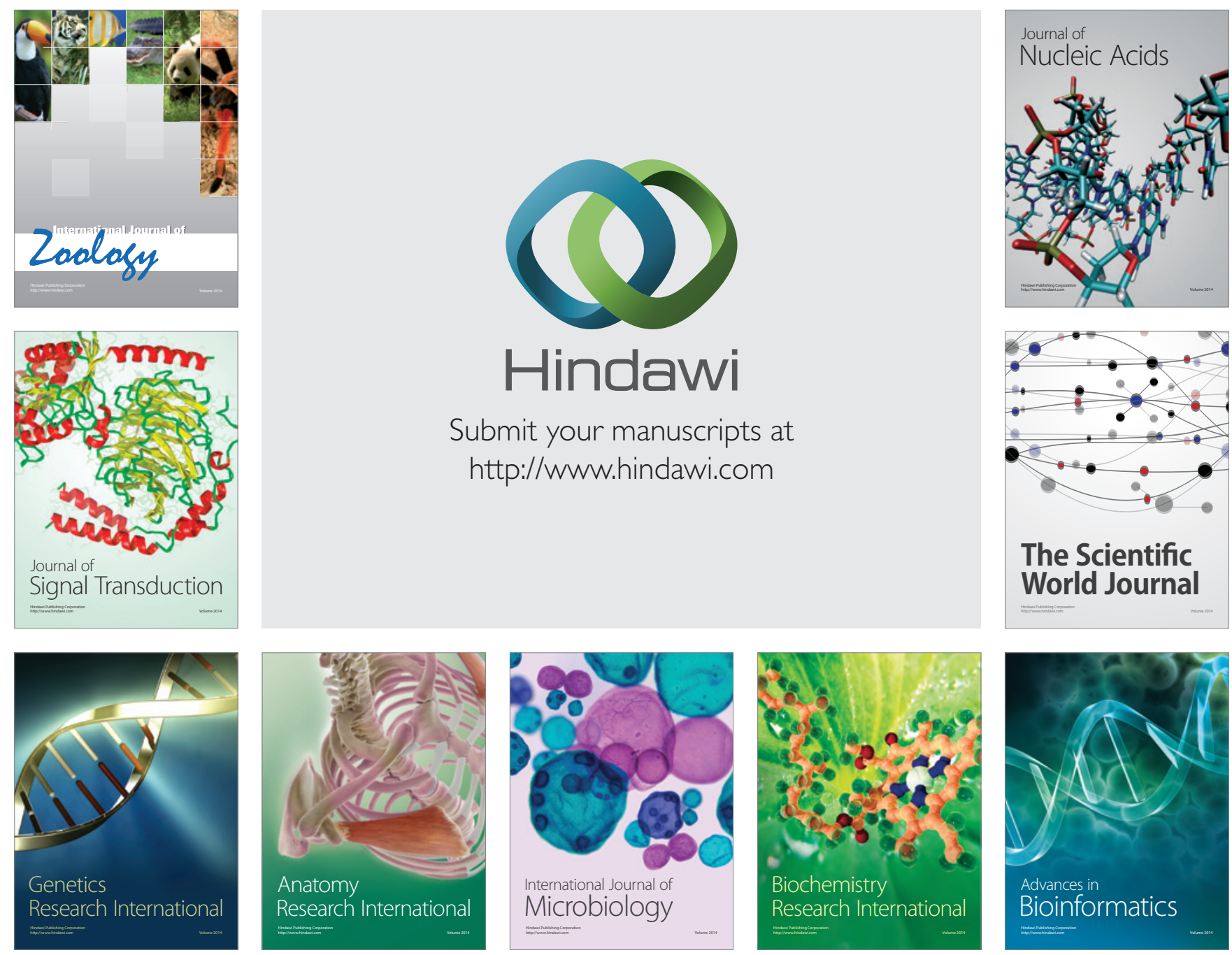

The Scientific World Journal
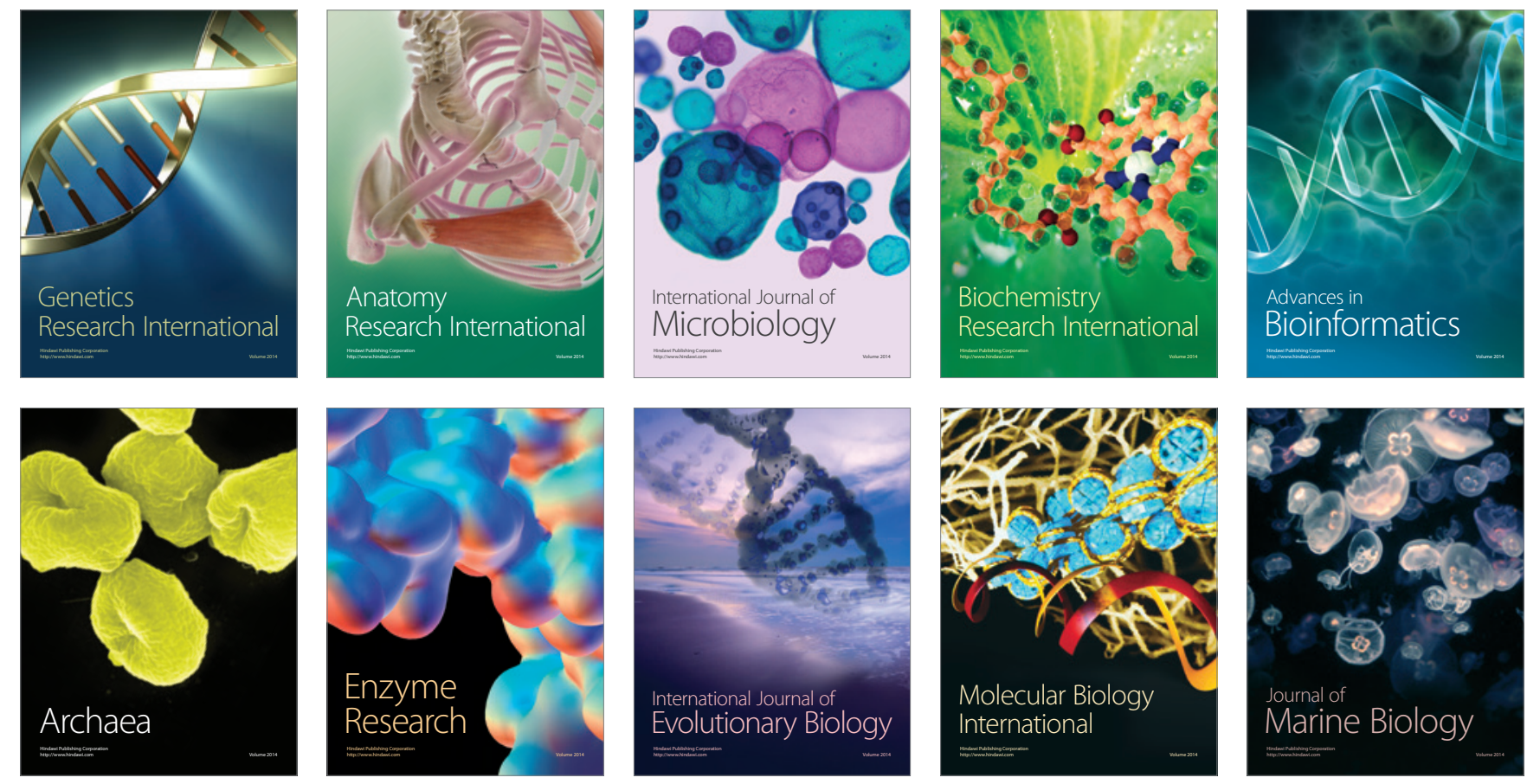